\title{
Conceptions historiques et identitaires des élèves francophones et anglophones de l'Ontario à l'époque post-11 septembre
}

\author{
Stéphane Lévesque (University of Western Ontario)
}

Résumé : Cet article traite du concept de «signification" ou sens donné aux événements historiques tel qu'employé par des élèves francophones et anglophones de l'Ontario à l'époque post-11 septembre. Il met en lumière la nécessité de mieux outiller les jeunes et les enseignants dans leur utilisation du passé canadien et dans leur apprentissage de la démarche historique en classe d'histoire ou d'études sociales.

\begin{abstract}
This article deals with the concept of « significance » or meaning given to historical events, as understood by Francophone and Anglophone students in Ontario in the post-September 11 period. The analysis reveals the necessity of providing youth and teachers with better means to their utilization of the Canadian past and in their learning of historical procedures in history or social studies classes.
\end{abstract}

\section{L’accélération de l'histoire}

Comme toute autre société moderne, la société canadienne est aux prises avec ce que Pierre Nora (2002) qualifie «d'accélération de l'histoire». Cette formule imagée signifie en clair que la succession des événements historiques, de même que leur amplitude, est dorénavant caractérisée non plus par la continuité mais bien par le changement de plus en plus rapide et étendu. Ce récent renversement de l'histoire, encore plus présent et perceptible depuis les attaques terroristes du 11 septembre 2001, a ni plus ni moins, pour Nora (2002, p. 4), « brisé l'unité du temps historique, la belle et simple linéarité, qui unissait au passé le présent et l'avenir ». Le passé n'est désormais plus garant de l'avenir. Il représente plutôt un boulet duquel on souhaite s'affranchir. Cette mouvance serait si profonde et si puissante que les identités collectives, notamment les identités minoritaires, se retrouvent littéralement renversées par la création de mémoires historiques à usages multiples (par exemple, politique, touristique, commercial). On peut penser que l'accélération de l'histoire pose de redoutables défis pour les jeunes qui tentent de s'intégrer à leur société, notamment ceux au sein des communautés minoritaires qui traditionnellement gardaient un rapport très étroit avec leur passé.

Face à cette situation, il est bon, voire nécessaire, de vérifier l'état des choses auprès des élèves canadiens eux-mêmes. Il m'apparaît tout à fait pertinent d'étudier ce que ces derniers savent, comprennent ou s'approprient de l'enseignement de l'histoire nationale dans un contexte particulier comme celui du Canada francophone et anglophone. Cette étude est d'autant plus importante 
que l'on soutient volontiers que les élèves des deux communautés linguistiques se voient enseigner des histoires nationales complètement différentes, engendrant, du même coup, la construction d'identités et de consciences historiques particulières. Comme le rappelle John Dickinson (1996, p. 148), «l'historiographie canadienne n'a jamais été unifiée et les deux traditions linguistiques sont aussi différentes l'une de l'autre que des historiographies étrangères. Aucune vue consensuelle n'a jamais existé qui pourrait réconcilier tous les Canadiens ${ }^{1}$. Face à ce constat, qu'en est-il des élèves des deux communautés linguistiques dans une province diversifiée comme celle de l'Ontario ?

\section{Qu'est-ce que la « signification » de l'histoire ?}

Un des concepts fondamentaux pour étudier l'histoire et construire des récits historiques est celui de la «signification» qui est donnée à l'histoire ou communément appelé en anglais l'historical significance. Puisque les historiens ne peuvent d'aucune façon étudier l'ensemble du passé collectif, ils doivent nécessairement être sélectifs dans leur démarche de questionnement et de recherche. Certains facteurs, événements, personnages ou phénomènes retiennent davantage leur intérêt. Tim Lomas (1990, p. 41) soutient que « l'on ne peut donner un sens à l'histoire sans le concept de signification. Pour avoir un sens, l'historien doit nécessairement faire une sélection, et cette sélection dépend de certains critères qui permettent de différencier ce qui est pertinent de ce qui ne l'est pas ». Pour Lomas, tous les historiens utilisent implicitement ou explicitement certains critères dans leur démarche historique. Mais quels sont ces critères? Pour quelle(s) raison(s) un événement historique devient-il signifiant, c'est-à-dire porteur d'une signification ou d'un sens particulier?

Cette recherche tente d'étudier la question. Quelle signification ou quel sens particulier les élèves des deux communautés linguistiques officielles de l'Ontario donnent-ils aux événements historiques? De manière plus spécifique, l'étude explore les mécanismes de sélection et d'attribution d'une certaine signification tels qu'employés par les élèves francophones et anglophones de cette province dans le contexte post-11 septembre. À ce jour, il n'existe pas de normes «officielles» pour déterminer la signification ou le sens attribuée au passé. Il est généralement convenu au sein de la communauté historique que les historiens professionnels doivent étudier scrupuleusement les sources et traces du passé en question s'ils veulent produire des connaissances dites « scientifiques »- bien que ce raisonnement implicite change de génération en génération et se trouve largement remis en question de nos jours avec les thèses postmodernistes à la Michel Foucault (voir Koselleck 2002).

\footnotetext{
${ }^{1}$ Dans cet article, nous avons traduit nous-même toutes les citations tirées de textes écrits en anglais.
}

32 Canadian and International Education Vol. 34 no. 1 - June 2005 
Par conséquent, il y a très peu de recherche historique sur ce concept fondamental, même en Angleterre où l'historical significance est partie intégrante des nouveaux programmes d'études en histoire. Robert Phillips (2002) soutient néanmoins qu'il existe certains facteurs qui guident les historiens dans leur choix et interprétations :

-L'importance: $\quad$ A trait à l'influence de l'événement pour ceux qui l'ont vécu, sans tenir compte si leur jugement s'est avéré juste dans le futur. Questions pouvant guider l'emploi du facteur d'importance : Qui fut affecté? L'événement étaitil important pour eux?

-L'ampleur: $\quad$ A trait à la profondeur et l'étendue de l'événement pour ceux qui furent affectés. Questions pouvant guider l'emploi du facteur d'ampleur: L'événement était-il superficiel ou profond? A-t-il véritablement affecté les gens?

- La quantité: $\quad$ A trait au nombre de personnes affectées (directement ou indirectement) par l'événement. Question pouvant guider l'emploi du facteur de quantité : L'événement a touché combien de personnes?

-Ladurée: $\quad$ A trait à l'étendue de l'événement dans le temps. Questions pouvant guider l'emploi du facteur de durée: L'événement fut-il long ou éphémère? Pendant combien de temps les gens furent-ils affectés?

-La pertinence: $\quad$ A trait à la construction et compréhension du sens donné à l'événement par les acteurs contemporains à partir des faits historiques. Questions pouvant guider l'emploi du facteur de pertinence: L'événement contribue-t-il à la compréhension du passé? L'événement sert-il de «leçon d'histoire »? L'événement a-t-il une symbolique particulière?

Traditionnellement, les événements historiques qui ont affecté un grand nombre de personnes (i.e., la quantité) ont reçu davantage d'intérêt (par exemple, une guerre mondiale par rapport à une rébellion). «Dans l'histoire, comme le soutient E.H. Carr (1986, p. 50), les chiffres comptent». Mais le fait qu'un grand nombre de personnes aient été affectées par un événement particulier ne garantit plus son application universelle au sein de la communauté historique. D'autres facteurs, tel la symbolique de l'événement, influent désormais sur la décision de l'historien.

Devant ce nouveau constat, Michael Stanford (1994, pp. 29-30) croit que la signification peut se schématiser selon deux dimensions complémentaires : historique (ou partant de la base), et contemporaine (ou partant du haut). La dimension historique se rapporte aux questions entourant ceux qui ont été 
affectés par les événements (importance, ampleur, quantité, durée) et la dimension contemporaine aux rapports qu'entretiennent les acteurs contemporains avec les événements passés (pertinence). J'ai déjà indiqué que certains événements semblent être plus signifiants que d'autres en raison de l'importance historique qu'ils ont eu pour ceux qui les ont directement vécus. Mais la signification, selon Stanford, doit également être examinée en fonction des circonstances contemporaines, c'est-à-dire en relation avec l'actuel. En effet, les historiens sont à la fois les "produits » et les «porte-parole» des sociétés auxquelles ils appartiennent. "L'historien, soutient Carr, n'est pas un spectateur, un V.I.P sur un podium observant un cortège, il fait partie du cortège... et son emplacement dans le cortège détermine en quelque sorte sa vision du passé » (1986, pp. 36-37). Faisant face à une quantité presque illimitée d'événements et de traces historiques, les historiens regardent le passé non seulement avec un œil sur le présent, mais également avec des questions, soucis et valeurs qui émanent d'un groupe sociétal particulier. Le rapport des historiens avec le passé est ainsi limité, ou à tout le moins influencé, par des circonstances qui leur sont contemporaines. À bien des égards, l'actuel désigne, voire prescrit, les domaines de recherche, les questions à poser et le matériel à investiguer.

Comme si tout cela n'était pas suffisant, beaucoup d'individus, incluant des historiens et des enseignants, sont également influencés par des considérations purement naïves ou utilitaristes, de sorte que le passé ne sert que de bouclier justificatif sans égard à la complexité des événements historiques ou de leur historicité. Ainsi, le sens attribué à un événement ne garantit en rien son fondement historique, même lorsque déterminé par les historiens, puisqu'ils sont eux-mêmes des acteurs empreints d'une certaine culture et temporalité. Le Canada est, à cet égard, un exemple étonnant.

En effet, depuis sa création, le pays a été profondément divisé par les deux communautés linguistiques d'appartenance. Plusieurs sujets (politique, religieux, constitutionnel, historiographique) qui ont été l'objet d'une variété d'études, attestent de cette division. En 1945, Walter Sage (1945, p. 4) faisait part, dans son discours à la Société historique du Canada, d'une situation qui lui semblait tout à fait accablante :

Depuis les jours de Michel Bibaud et François-Xavier Garneau à ceux du sénateur Thomas Chapais, et de l'abbé Groulx, il y a deux traditions historiographiques au Canada, celle de langue française et celle d'expression anglaise. Malheureusement, la relation entre ces deux groupes est loin d'être achevée. En fait, il semble qu'il y ait à l'occasion plus de rupture que de liaison entre les deux. La barrière linguistique demeure une réalité.

Les propos de Sage étaient loin d'exagérer la réalité des « deux solitudes ». Au plan scolaire, la formation pédagogique des maîtres, de même que les volumes et les manuels scolaires canadiens anglais, ont historiquement laissé entendre 
que la sélection et l'attribution d'un sens historique devait s'effectuer en fonction de critères tels que la race (blanche), la langue (anglaise) et/ou le statut social (bourgeoisie, noblesse, royauté). Tout naturellement, les Canadiens français, et plus particulièrement le Clergé catholique en charge de l'éducation des francophones, ont refusé une telle hégémonie historiographique pour des raisons politiques et culturelles évidentes. En réponse, ils se sont concentrés sur la transmission d'autres événements et personnages historiques du passé collectif qui mettaient davantage en lumière la survivance du peuple canadien français. Comme le rapporte Gérard Bouchard (2000, p. 81), « un fil conducteur s'est maintenu [au Canada français] : celui d'une francophonie qui, dans des conditions très difficiles, a tant bien que mal assuré sa reproduction en Amérique et s'en est fait une vocation ». De nos jours, on peut soutenir que malgré une meilleure reconnaissance du «fait français » au Canada et une plus grande diversité et mobilité chez les historiens canadiens, cette division qui a marqué le développement et la progression du pays est toujours présente (voir également Cardin, 2003).

Mais les Canadiens ne sont pas seuls sur ce terrain historique contesté. De récentes études (Barton et Levstik, 1998 ; Epstein, 1998 ; Seixas, 1994 ; Yeager, Foster et Greer, 2002) indiquent que le concept de "signification » est non seulement problématique mais revendiqué à plusieurs fins. "Les normes régissant la signification, comme le soutient Peter Seixas (1997, p. 22), n'émanent pas uniquement des événements historiques mais aussi et surtout des valeurs et questionnements de ceux qui étudient le passé ». En ce sens, les élèves, les enseignants, les citoyens, de même les historiens eux-mêmes, interprètent le passé collectif à partir de leur propre modèle interprétatif construit sous l'influence de diverses forces politiques, culturelles, linguistiques, patrimoniales, populaires, et aussi éducatives.

Étant confronté à cette situation complexe, caractérisée par un enchevêtrement entre la culture et mémoire collective et la discipline historique, j'ai donc décidé de réévaluer la notion même de signification en distinguant différentes communautés d'appartenance qui caractérisent les domaines à l'intérieur desquels les membres (historiens, enseignants, élèves, etc.) définissent leur signification de l'historique. En règle générale, les historiens professionnels font leur sélection historique en utilisant un ensemble de critères disciplinaires présentés précédemment (importance, ampleur, quantité, durée et pertinence). Toutefois, ces critères couramment employés en historiographie ne pas très bien articulés ou utilisés en dehors de la communauté historique. Le résultat a donc été le développement et l'utilisation d'autres critères; une sorte de " bricolage » de normes populaires largement déterminées par la mémoire collective. Loin de favoriser le développement de la conscience historique, ces normes visent plutôt l'avancement de l'identité et de la conscience patrimoniale. De manière plus spécifique, ces normes peuvent être définies comme instruments contemporains ayant pour but d'attribuer une " signification de la mémoire » au passé collectif. L'élaboration de ces normes est importante car elle aide à mieux comprendre 
comment et pourquoi les individus, notamment au sein du système scolaire, établissent un rapport avec le passé. Pour les fins de cette recherche, j'ai divisé ces normes en trois catégories :

- Les intérêts particuliers : Signification historique attribuée en fonction de rapports personnels, familiaux, religieux, culturels, ou ancestraux avec les événement historiques (par exemple, cet événement est important car ma famille y était);

- La signification symbolique : Signification historique attribuée en fonction de critères patriotiques ou collectif (par exemple, la fête nationale est importante pour moi);

- Les leçons contemporaines : Signification historique attribuée en fonction d'analogies simplistes servant à guider les actions présentes ou futures, habituellement pour éviter les «erreurs» du passé (par exemple, la Grande dépression nous enseigne qu'il faut éviter la stipulation financière).

Ces normes ou critères de signification de la mémoire, largement employés par le public et la communauté éducative, ajoutés aux quatre critères employés par les historiens professionnels, démontrent toute la complexité quant à la sélection et attribution d'un sens particulier pour les élèves (voir Cercadillo, 2001, pour un ensemble de normes similaires).

\section{Qu'en pensent les élèves ?}

Dans le but d'en connaitre davantage sur la signification telle que perçue par les élèves canadiens, cette étude comparative explore comment les élèves francophones et anglophones du niveau secondaire de l'Ontario utilisent le concept de signification dans le cadre de l'enseignement de l'histoire canadienne. Tous les élèves volontaires, pour chaque groupe linguistique inscrits en histoire, 78 élèves de niveau secondaire ( 38 francophones et 40 anglophones) du sud-ouest ontarien, ont pu participer à l'étude.

L'étude est basée sur l'analyse de réponses à un questionnaire (français et anglais) comportant deux étapes : (1) choisir parmi la liste des 24 événements présélectionnés du passé canadien les cinq événements plus importants et (2) expliquer les raisons de cette sélection dans l'espace questionnaire prévu à cet effet. Les événements, de même que les représentations visuelles incluses (par exemple, une photographie) dans le questionnaire, proviennent d'une analyse de contenu des programmes francophones et anglophones de l'Ontario approuvés pour l'enseignement de l'histoire du Canada. Il est important de noter que les élèves pouvaient également faire référence un événement historique complémentaire, non inclus dans le questionnaire. À part quelques exceptions 
(par ex. : Le projet Avro-Arrow), la grande majorité des élèves francophones et anglophones s'en sont tenus à la liste offerte. Pour les fins d'analyse, seuls les événements inclus dans le questionnaire sont présentés dans cet article.

TABLEAU 1

Sélection des événements historiques du passé canadien par groupe linguistique

\begin{tabular}{|l|c|c|c|}
\hline (n=78) & Total & Total & Total \\
\hline Événements historiques & & Francophone & Anglophone \\
\hline Première guerre mondiale 1914-1918 & $\mathbf{3 7}$ & $\mathbf{1 7}$ & $\mathbf{2 0}$ \\
\hline Drapeau canadien, 1965 & $\mathbf{3 2}$ & $\mathbf{1 5}$ & $\mathbf{1 7}$ \\
\hline Confédération, 1867 & $\mathbf{3 2}$ & $\mathbf{1 2}$ & $\mathbf{2 0}$ \\
\hline Droits des femmes, 1920s & $\mathbf{3 0}$ & $\mathbf{1 7}$ & $\mathbf{1 2}$ \\
\hline Guerre de 1812 & $\mathbf{2 7}$ & $\mathbf{3}$ & $\mathbf{2 4}$ \\
\hline 11 septembre 2001 & $\mathbf{2 7}$ & $\mathbf{1 8}$ & $\mathbf{9}$ \\
\hline Deuxième guerre 1939-1945 & $\mathbf{2 6}$ & $\mathbf{1 6}$ & $\mathbf{1 0}$ \\
\hline Chemin de fer clandestin 18402 & $\mathbf{2 1}$ & $\mathbf{8}$ & $\mathbf{1 3}$ \\
\hline Grande Dépression, 1930s & $\mathbf{2 0}$ & $\mathbf{1 0}$ & $\mathbf{1 0}$ \\
\hline Résistance franco-ontarienne, 1916 & $\mathbf{1 9}$ & $\mathbf{1 7}$ & $\mathbf{2}$ \\
\hline Canada et le maintien de la paix 1956-1957 & $\mathbf{1 8}$ & $\mathbf{4}$ & $\mathbf{1 4}$ \\
\hline Chute de la Nouvelle France, 1759 & $\mathbf{1 4}$ & $\mathbf{4}$ & $\mathbf{1 0}$ \\
\hline Époque de la découverte 16e siècle & $\mathbf{1 4}$ & $\mathbf{4}$ & $\mathbf{1 0}$ \\
\hline Référendum de 1995 & $\mathbf{1 2}$ & $\mathbf{1 0}$ & $\mathbf{2}$ \\
\hline Acte de Québec, 1774 & $\mathbf{9}$ & $\mathbf{2}$ & $\mathbf{7}$ \\
\hline Rapatriement de la Constitution, 1982 & $\mathbf{8}$ & $\mathbf{6}$ & $\mathbf{2}$ \\
\hline Crise d'Oka, 1990 & $\mathbf{5}$ & $\mathbf{4}$ & $\mathbf{1}$ \\
\hline Accord de libre échange, 1988 & $\mathbf{5}$ & $\mathbf{2}$ & $\mathbf{3}$ \\
\hline Coloniser l'ouest canadien, fin 19e siècle & $\mathbf{5}$ & $\mathbf{1}$ & $\mathbf{4}$ \\
\hline Rébellion de 1837-1838 & $\mathbf{5}$ & $\mathbf{2}$ & $\mathbf{3}$ \\
\hline Migration des loyalistes 1776-1783 & $\mathbf{4}$ & $\mathbf{2}$ & $\mathbf{2}$ \\
\hline Rébellion de la Rivière Rouge, 1869-1870 & $\mathbf{3}$ & $\mathbf{1}$ & $\mathbf{2}$ \\
\hline Révolution tranquille, 1960s & $\mathbf{1}$ & $\mathbf{2}$ \\
\hline Crise d'octobre, 1970 & $\mathbf{1}$ & $\mathbf{0}$ \\
\hline
\end{tabular}

Les résultats de la recherche démontrent que les événements historiques les plus signifiants pour les participants ont trait à la création du Canada (Confédération, 1867), à la participation de la colonie/pays aux conflits internationaux (Guerre de 1812, Première Guerre mondiale), à l'obtention des droits fondamentaux par les femmes (Droits des femmes, années 1920) et à l'adoption du drapeau canadien (1965). L'événement le plus récent (Attaques terroristes du 11 septembre 2001) apparaît au 5e rang, suivi d'une série d'événements historiques entourant les guerres et conflits, les mouvements sociaux, les enjeux économiques et les relations entre francophones et anglophones (voir Tableau 
1). Les résultats permettent également de voir l'étendue historique des événements choisis (du 16e au 21e siècle). Il ne semble donc pas, comme le laissent prétendre certains, qu'il y ait une sorte de prédisposition des jeunes aux événements d'actualité.

L'analyse des résultats par communauté linguistique offre néanmoins des choix plus variés et distincts. Bien que les deux premiers événements retenus (Première Guerre mondiale et Drapeau canadien) soient comparables, d'autres événements historiques mettent en relief l'influence de la langue/culture dans le processus de sélection et d'attribution d'une signification particulière. La Guerre de 1812, la Résistance franco-ontarienne, le Canada et le maintien de la paix et le Référendum de 1995 furent traités différemment par les élèves francophones et anglophones. À titre d'exemple, la Guerre de 1812 n'a retenu l'attention que de trois élèves francophones comparativement à 24 anglophones. À l'inverse, 17 élèves francophones ont sélectionné la Résistance franco-ontarienne contrairement à seulement deux élèves anglophones.

Mais les différences entre membres de communautés linguistiques ne s'arrêtent pas là. Les justifications offertes par les élèves permettent de discerner l'influence prépondérante de certains facteurs liés au sens particulier donné au passé (voir Tableaux 2 et 3). Pour l'ensemble des unités syntaxiques codées pour chaque groupe respectif, les élèves anglophones ont davantage fait référence aux facteurs disciplinaires $(65 \%)$ dans leurs explications que leurs collègues francophones (59\%). À l'inverse, les élèves francophones ont utilisés plus d'éléments faisant référence à la signification de la mémoire $(41 \%)$ que ceux du coté anglophone (35\%).

TABLEAU 2

Fréquence des réponses des élèves en fonction des critères disciplinaires (par groupe linguistique)

\begin{tabular}{|l|c|c|c|c|c|}
\hline \multicolumn{1}{|c|}{ Critères } & & \multicolumn{2}{c|}{ Francophone } & \multicolumn{2}{c|}{ Anglophone } \\
\hline & Grand total & Total & Pourcent & Total & Pourcent \\
\hline Importance & 212 & 79 & 41 & 133 & 41 \\
\hline Quantité & 74 & 39 & 20 & 35 & 11 \\
\hline Durée & 95 & 52 & 27 & 43 & 13 \\
\hline Pertinence & 135 & 24 & 12 & 111 & 35 \\
\hline Total & 516 & 194 & 100 & 312 & 100 \\
\hline
\end{tabular}


TABLEAU 3

Fréquence des réponses des élèves en fonction des critères de mémoire (par groupe linguistique)

\begin{tabular}{|l|c|c|c|c|c|}
\hline \multicolumn{1}{|c|}{ Critères } & & \multicolumn{2}{c|}{ Francophone } & \multicolumn{2}{c|}{ Anglophone } \\
\hline & Grand total & Total & Pourcent & Total & Pourcent \\
\hline Signification symbolique & 153 & 48 & 36 & 105 & 59 \\
\hline Intérêts particuliers & 87 & 68 & 50 & 19 & 11 \\
\hline Leçons contemporaines & 72 & 19 & 14 & 53 & 30 \\
\hline Total & 312 & 135 & 100 & 177 & 100 \\
\hline
\end{tabular}

En termes concrets, les élèves anglophones n'ont pas hésité à se référer aux facteurs d' " importance » et de " pertinence » (comme facteurs disciplinaires) et à la "signification symbolique » (pour la signification dite de la mémoire). Chez les élèves francophones, ce sont plutôt les facteurs de "durée» et d' « importance» (comme facteurs disciplinaires), de même que les «intérêts particuliers » qui semblent avoir joué un plus grand rôle dans leurs justifications.

\section{Que peut-on en conclure ?}

Que peut-on inférer à partir de cette étude ? Tout d'abord, il est entièrement erroné de prétendre que les jeunes Canadiens ne connaissent rien de leur passé collectif. On peut bien s'offusquer de leurs connaissances factuelles souvent limitées, mais il n'en demeure pas moins que les élèves attachent une importance particulière aux événements marquants du passé canadien. Cette importance est telle que plusieurs n'hésitent pas à considérer des événements historiques, comme la Guerre de 1812, plus signifiants que des événements d'actualité auxquels ils peuvent rattacher un certain vécu ou même des souvenirs personnels, tel les attaques du 11 septembre 2001.

L'étude permet également de mettre en lumière l'influence notable, mais souvent méconnue, de la culture/langue d'enseignement en classe d'histoire. On savait depuis longtemps que les jeunes Québécois ne partageaient pas nécessairement les mêmes référents historiques que leurs compatriotes anglophones; distinction que l'on pourrait attribuer, avec raison, au système provincial d'éducation. Or, cette étude démontre que les jeunes (même en milieu minoritaire) entretiennent un rapport avec l'histoire nationale qui ne se limite pas seulement au monde scolaire. En effet, les membres des deux communautés linguistiques de l'Ontario suivent, grosso modo, les mêmes programmes d'histoire du Canada avec les mêmes volumes scolaires (traduits de l'anglais au 
français). De plus, le concept historique de «signification» n'est pas au programme de la province. En ce sens, on peut donc croire que le milieu dans lequel vivent et se développent les jeunes francophones et anglophones exerce une influence considérable sur leur sélection et leur justification historique. Ainsi, le plus grand nombre de références aux « intérêts particuliers » chez les francophones peut témoigner du rôle que joue la culture minoritaire francophone en Ontario. Cette culture s'est historiquement protégée contre l'assimilation causée par l'influence de l'élite canadienne anglaise et britannique dont les intérêts sont largement représentés dans les documents pédagogiques et la culture populaire. À l'opposé, les élèves anglophones n'ont pas hésité à se référer à des événements historiques qui représentaient une "signification symbolique » ou revêtaient une certaine "pertinence». Étant membres de la culture dominante, ils ont tout naturellement mis l'accent sur des symboles nationaux ou faits historiques perçus comme positifs au sein de leur communauté majoritaire.

Bref, on peut soutenir en conclusion qu'en l'absence d'une conception éclairée, voire plus disciplinaire, de la notion de «signification » en classe d'histoire, les élèves s'en remettent tout naturellement à ce que Jocelyn Létourneau (2004, p. 347) appelle le "régime historial socialement accepté». Dans un monde caractérisé par l'accélération de l'histoire et où l'historien doit dorénavant partager son rôle de production (et de diffusion) des connaissances avec les enseignants, les législateurs, les musées, les médias, et même Hollywood, il est impératif que les jeunes soient guidés dans leur processus de sélection et d'attribution d'un sens particulier au passé collectif. Cette étude tend à démontrer qu'il faudrait mieux outiller les élèves et les enseignants en classe d'histoire. Si l'histoire scolaire, comme le soutient Christian Laville (2002, p. 6), a été traditionnellement employée à titre "d'éducation civique nationale » afin de confirmer et légitimer l'État-nation, la démocratie moderne nécessite désormais l'apprentissage de capacités intellectuelles et disciplinaires qui visent à former des citoyens compétents et responsables - tout en étant bien ancrés dans leur communauté d'appartenance. En ce sens, l'histoire scolaire doit trouver un juste équilibre entre le « devoir de mémoires historiques partagées » et les besoins de la « conscience historique ».

\section{Notes bibliographiques}

Barton, K., \& Levstik, L. (1998). 'It wasn't a good part of history': National identity and students' explanations of historical significance. Teachers College Record, 99, 478-513.

Bouchard, G. (2000). Genèse des nations et cultures du nouveau monde: essai d'histoire comparée. Montréal : Éditions Boréal.

Cardin, J. (2003) Essai sur la mise on récit de l'expérience historique canadienne. Thèmes canadiens, Octobre, 16-21.

Carr, E. H. (1986). What is History? London : Penguin.

40 Canadian and International Education Vol. 34 no. 1 - June 2005 
Cercadillo, L. (2001). Significance in history: Students' ideas in England and Spain. In A. Dickinson, P. Gordon \& P. Lee (eds), Raising standards in history education: International Review of History Education, 3, 116-145.

Dickinson, J. (1996). Canadian historians - Agents of unity of disunity. Journal of Canadian Studies, 31, 148.

Epstein, T. (1998). Deconstructing differences in African-American and EuropeanAmerican adolescents' perspectives on U.S. History. Curriculum Inquiry, 28 : 397-423.

Koselleck, R. (2002). The practice of conceptual history: Timing history, spacing concept. Stanford : Stanford University Press.

Létourneau, J. (2004). Mémoire et récit de l'aventure historique du Québec chez les jeunes Québécois d'héritage canadien-français: coup de sonde, amorce d'analyse des résultats, questionnements. The Canadian Historical Review, 84, 325-356.

Laville, C. (2002). Que (re)viendrait faire la mémoire dans l'enseignement de l'histoire? Encounters on education/Rencontres sur l'éducation/Encuentros sobre educación, 3, 5-25.

Lomas, T. (1990). Teaching and assessing historical understanding. London: The Historical Association.

Nora, P. (2002). L'avènement mondial de mémoire. Eurozine, 19 mars. Disponible : http://www.eurozine.com.

Phillips, R. (2002). Historical significance: The forgotten 'key concept'? Teaching History, 106, 14-19.

Sage, W. (1945). Where stands Canadian history? Allocution présidentielle au congrès annuelle de la Société historique du Canada. Disponible: http://www.chashc.ca/bilingue/addresses/ 1945.htm.

Seixas, P. (1994). Students' understanding of historical significance. Theory and Research in Social Education, 22, 281-304.

Seixas, P. (1997). Mapping the terrain of historical significance. Social Education, 61, 22-27.

Stanford, M. (1988). A companion to the study of history. London : Blackwell.

Yeager, E., Foster, S. \& Greer, J. (2002). How eighth graders in England and the United States view historical significance. The Elementary School Journal, 13, 199-219. 